INPLASY

PROTOCOL

To cite: Thapa et al. A metaanalysis on effect of complex training on sprint, jump and change of direction ability of soccer players. Inplasy protocol 202090079. doi: 10.37766/inplasy2020.9.0079

Received: 21 September 2020

Published: 21 September 2020

Corresponding author:

Rohit Kumar Thapa

rohitthapa04@gmail.com

Author Affiliation:

Lakshmibai National Institute of Physical Education

Support: Self.

Review Stage at time of this submission: Data analysis.

Conflicts of interest: None.

\section{A meta-analysis on effect of complex training on sprint, jump and change of direction ability of soccer players}

\author{
Thapa, RK¹; Ramirez-Campillo, R²; Lum, D³.
}

Review question / Objective: Whether a complex training intervention of a minimum four weeks duration improves the sprint, jump, or change of direction performance in soccer players?

Condition being studied: Complex training has recently been a widely studied training method to improve performance in athletes. It utilizes both strength and plyometric activities in a single session. Both strength training and plyometric training has been shown to improve performance in soccer players. But conducting training sessions of strength and plyometric in a micro-cycle with matches becomes challenging for strength and conditioning coaches, as soccer coaches want more technical and tactical training time. Using complex training sessions may benefit the players with both the strength and plyometric sessions. This meta-analysis is conducted to find the pooled effect of various studies in complex training on soccer players.

INPLASY registration number: This protocol was registered with the International Platform of Registered Systematic Review and Meta-Analysis Protocols (INPLASY) on 21 September 2020 and was last updated on 21 September 2020 (registration number INPLASY202090079).

\section{INTRODUCTION}

Review question / Objective: Whether a complex training intervention of a minimum four weeks duration improves the sprint, jump, or change of direction performance in soccer players?
Rationale: Soccer is a sport that requires high power output during sprinting, jumping, and changing direction. Complex training (CT) has been found effective in improving the physical abilities requiring high-power output. The lack of meta- 
analysis studies on CT targeted at soccer players' performance, it is appropriate to analyze studies focused on describing these effects.

Condition being studied: Complex training has recently been a widely studied training method to improve performance in athletes. It utilizes both strength and plyometric activities in a single session. Both strength training and plyometric training has been shown to improve performance in soccer players. But conducting training sessions of strength and plyometric in a micro-cycle with matches becomes challenging for strength and conditioning coaches, as soccer coaches want more technical and tactical training time. Using complex training sessions may benefit the players with both the strength and plyometric sessions. This meta-analysis is conducted to find the pooled effect of various studies in complex training on soccer players.

\section{METHODS}

Search strategy: The following combination of keywords was used in the search databases, "complex training" or "contrast training" or "combination of strength training and plyometrics" and "soccer" or "football." An example of the search strategy used in PubMed was: ((( (complex training) OR contrast training) OR (combination of strength training and plyometrics)) AND soccer) OR football. Relevant articles' reference list was examined to find out possible articles for inclusion in the meta-analysis.

Participant or population: Cohorts of healthy soccer players, with no restrictions for age or sex.

Intervention: A complex training intervention of at least 4 weeks, which utilizes a heavy load exercise followed by a low load exercise, set for set.

Comparator: A control group or strength training group of soccer players.
Study designs to be included: Randomized and controlled trials.

Eligibility criteria: The studies were included if they met the following criteria: (i) subjects in the study were soccer players involved in at least four weeks duration of the intervention program; (ii) at least one group participated in complex training intervention with lower body exercises; (iii) consist of active control group or traditional strength training group (iv) the outcome variables were reported and included sprint, jump or change of direction ability assessments, since this physical measures have been instrumental in overall performance during a soccer game (Faude et al 2012; Castagna et al., 2003) and can be a differentiating factor between an amateur and an elite player. (v) the study design consists of controlled trials.

Information sources: Electronic database searches were conducted using PubMed and Google scholar. Following the formal systematic searches, additional handsearches were conducted.

Main outcome(s): Physical fitness outcomes such as sprinting, jumping, and changing of direction ability are an appropriate measure of muscular power, requiring a fast transition between the eccentric and concentric phases of muscle action. Such outcome measures also present a very high test-retest reliability, and were therefore chosen for the metaanalysis. Means and standard deviation of pre to post measurements and post measurements were used for the analysis.

Data management: Data were extracted from included articles using a form created in Microsoft Excel (Microsoft Corporation, Redmond, WA, USA). In cases where data required were not clearly or completely reported, the authors of the articles were contacted for clarification.

Quality assessment / Risk of bias analysis: The Tool for the assEssment of Study qualiTy and reporting in EXercise (TESTEX) was used to assess the quality of studies 
included in this meta-analysis The TESTEX comprises 12 methodological quality assessment criteria. The maximum score a study can obtain is $\mathbf{1 5}$ points, with higher ratings reflecting better study quality and reporting.

Strategy of data synthesis: For analysis and interpretation of results, meta-analyses were conducted if at least two studies provided data for the same parameter. Meta-analytical comparisons were carried out in the Review Manager program (version 5.3). The inverse variance randomeffects model for meta-analyses was used. The differences were expressed as standard mean difference (SMD) and their 95\% confidence intervals (Cls). The effect sizes (SMD) were estimated with threshold values using the following scale: 0.6-1.2, moderate; $>1.2-2.0$, large, $>2.0-4.0$, very large; $>4.0$, extremely large. To gauge the degree of heterogeneity amongst the included studies, the 12 statistic was referred to low, moderate, and high levels of heterogeneity correspond to 12 values of 25,50 , and $75 \%$, respectively.

Subgroup analysis: Subgroup analyses were performed on the pre-post data of variables that could have influenced the outcome after CT interventions, with median values of continues variables used as cut-off values for grouping (age and duration). The subgroup chosen for analysis was based on age ( $\geq 18$ years vs $<18$ years), competition level (elite vs amateur), and duration of complex training intervention ( $\geq 8$ weeks vs $<8$ weeks). The analysis was conducted when there were at least 1 study in the subgroups. Subjects in this meta-analysis were considered as elite if they practiced soccer professionally or semi-professionally and amateurs if they practiced soccer at an amateur club, college, or university as reported in the study.

Sensibility analysis: Sensibility analysis was not conducted.

Language: Only articles published in English language were considered.
Country(ies) involved: India; Chile; Singapore.

Keywords: contrast training, football, power, plyometric, post-activation performance enhancement.

Contributions of each author:

Author 1 - Rohit Kumar Thapa.

Author 2 - Rodrigo Ramirez-Campillo.

Author 3 - Danny Lum. 